\title{
ASYMPTOTIC EXPANSIONS FOR DISTRIBUTIONS OF COMPOUND SUMS OF RANDOM VARIABLES WITH RAPIDLY VARYING SUBEXPONENTIAL DISTRIBUTION
}

\author{
PH. BARBE, ${ }^{*}$ CNRS \\ W. P. McCORMICK $* * * * *$ AND \\ C. ZHANG, ${ }^{* *}$ University of Georgia
}

\begin{abstract}
We derive an asymptotic expansion for the distribution of a compound sum of independent random variables, all having the same rapidly varying subexponential distribution. The examples of a Poisson and geometric number of summands serve as an illustration of the main result. Complete calculations are done for a Weibull distribution, with which we derive, as examples and without any difficulties, seven-term expansions.
\end{abstract}

Keywords: Asymptotic expansion; convolution; tail area approximation; regular variation; subexponential distribution

2000 Mathematics Subject Classification: Primary 60F99

Secondary 60K05; 60G50; 41A60

\section{Introduction}

In this paper we construct asymptotic expansions for the tail area of a compound sum, when the distribution of the summands belongs to a class of rapidly varying subexponential distributions. To be more precise, let $X_{i}, i \geq 1$, be a sequence of independent random variables, all having the same distribution, $F$. For any positive integer $n$ the distribution of the partial sums $S_{n}=X_{1}+\cdots+X_{n}$ is the $n$-fold convolution $F^{\star n}$. We set $S_{0}=0$ and therefore $F^{\star 0}$ is defined as the distribution of the point mass at the origin. Let $N$ be a nonnegative integer-valued random variable, independent of the $X_{i}$ s. We consider the distribution $G$ of the compound sum $S_{N}$, that is $\mathrm{E} F^{\star N}$, and we seek an asymptotic expansion for its tail area $\bar{G}=1-G$.

First-order asymptotic results for $\bar{G}$ have been obtained by Embrechts et al. (1979), Cline (1987), and Embrechts (1985). A second-order formula may be found in Grübel (1987) and Omey and Willekens (1987).

Compound sums or subordinated distributions arise as distributions of interest in several stochastic models. In insurance risk theory, it models the total claim amount. For a discussion of issues related to random sums and insurance risk, we refer the reader to Embrechts et al. (1997), Asmussen (1997), and Goldie and Klüppelberg (1998). Compound sums also appear in queueing theory, in connection with the stationary distribution of waiting times in the GI/G/1 queue. The connection here is not as direct as in the insurance risk model in that it is derived from an analysis of ladder heights for transient random walks; see, for example,

Received 27 September 2006; revision received 14 May 2007.

* Postal address: 90 rue de Vaugirard, 75006 Paris, France.

** Postal address: Department of Statistics, University of Georgia, Athens, GA 30602, USA.

*** Email address: bill@stat.uga.edu 
Asmussen (1987, p. 80), Feller (1971, p. 396), and Pakes (1975). Another common way in which this model occurs is through the solution to a transient renewal equation. An example of this occurs in branching processes, where we obtain a geometric-compound sum in the analysis of the mean number of particles alive at a given time in an age-dependent subcritical process; see Athreya and Ney (1972, p. 151). We refer the reader to Feller (1971, Chapter XI) for a discussion of transient renewal theory. For further applications of subexponentiality in transient renewal theory, we refer the reader to Teugels (1975) and Embrechts and Goldie (1982).

We conclude this introduction by discussing two technical points. While the technique developed in this paper is applied in a more restrictive setting than that promoted by Grübel (1987), it appears to lead to results in a form more suitable for computation. Moreover, in his improvement of the so-called Banach algebra method, Grübel (1987) pointed out that 'in principle this should lead to arbitrarily fine expansions'; to the authors' knowledge, the Banach algebra method has yet to be used to produce such explicit fine results comparable to the one we present here for the problem at hand.

A second technical aspect of the current paper is that for regularly varying tails, Barbe and McCormick (2004) obtained expansions for compound sums based on an expansion for weighted convolutions with an estimate of the remainder term. In contrast to this, for rapidly varying subexponential tails, Barbe and McCormick (2005) did not provide explicit bounds, thereby precluding use of the method from Barbe and McCormick (2004) to obtain applied probability applications to be carried over to the present setting. Thus, one goal of the current paper is to obtain compound sum tail expansions in the rapidly varying subexponential setting with less information than was available in the regularly varying setting in Barbe and McCormick (2004).

\section{Main results}

If it exists ultimately, the hazard rate $h=F^{\prime} / \bar{F}$ yields the representation of the distribution function $F$ as

$$
\bar{F}(t)=\bar{F}\left(t_{0}\right) \exp \left(-\int_{t_{0}}^{t} h(u) \mathrm{d} u\right)
$$

ultimately. We write 'Id' for the identity function on $\mathbb{R}$; for any positive real number $r$, the function $\operatorname{Id}^{r}$ maps $t$ to $t^{r}$. From the representation of $\bar{F}$ in terms of its hazard rate, we see that if $h \sim \alpha$ / Id at $\infty$, then $\bar{F}$ is regularly varying with index $-\alpha$. If $\lim _{t \rightarrow \infty} h(t)=\alpha$, then $\bar{F}(t)=\mathrm{e}^{-\alpha t(1+o(1))}$ has tail behavior close to that of an exponential distribution. Since we are interested in rapidly varying subexponential tails, it is natural to consider hazard rates such that

$$
\begin{gathered}
h \text { is regularly varying, } \\
\lim _{t \rightarrow \infty} t h(t)=+\infty, \quad \text { and } \lim _{t \rightarrow \infty} h(t)=0 .
\end{gathered}
$$

In order to be not too close to the Pareto-type distributions, we will strengthen this assumption by requiring that

$$
\liminf _{t \rightarrow \infty} \frac{t h(t)}{\log t}>0 .
$$

This excludes distributions with tail $\mathrm{e}^{-(\log t)^{a}}$ with $a<2$, but includes those for which $a \geq 2$. The class of distribution functions satisfying these conditions includes the subexponential Weibull ones as well as distributions transformationally related to the normal, such as the log-normal and Johnson's $S_{U}$ system; see Stuart and Ord (1994, Section 6.35). Specific purpose distributions that fall within the class includes Benktander type I and II; see 
Beirlant et al. (1996, Section 1.4). Our class also includes distribution functions with tails of the form $t^{\beta} \mathrm{e}^{-t^{\alpha}}$, with $\beta$ arbitrary real and $\alpha$ positive and less than 1 , which have been considered in the extreme value literature; see Rootzén (1986) and Davis and Resnick (1988).

For modeling tail behavior for distributions with Weibull-like tails, a mean excess plot, i.e. a plot of $\mathrm{E}(X-x \mid X>x)$, is revealing; see Beirlant et al. (1995). Under a mild regularity condition for the class of distribution functions considered in this paper, it can be established that the conditional variance function satisfies $\operatorname{var}(X \mid X>x) \sim 2 / h^{2}(x)$ as $x \rightarrow \infty$, thus allowing us to examine the adequacy of the model based on the data.

As observed in Barbe and McCormick (2004), (2005), smoothness is a key requirement to obtain asymptotic expansions. For our purposes, a good class of regularly varying functions are the smoothly varying ones of a given order, whose definition we now recall.

Definition 2.1. A function $h$ is smoothly varying of index $\alpha$ and order $m$ if it is ultimately $m$-times continuously differentiable and its $m$ th derivative is regularly varying of index $\alpha-m$.

Clearly, if the hazard rate is $m$ times differentiable, then the tail function $\bar{F}$ can be differentiated $m+1$ times.

The next notation we need to introduce pertains to the Laplace characters. We write D for the derivation operator; that is, if $g$ is differentiable then $\mathrm{D} g$ is its derivative. As is customary, we define $\mathrm{D}^{0}$ to be the identity and, for any positive integer $i$, we define $\mathrm{D}^{i}$ by induction as $\mathrm{DD}^{i-1}$.

We write $\mu_{F, i}$ for the $i$ th moment of $F$.

Definition 2.2. (Barbe and McCormick (2004).) Let $F$ be a distribution function having at least $m$ moments. Its Laplace character of order $m$ is the differential operator

$$
L_{F, m}=\sum_{0 \leq i \leq m} \frac{(-1)^{i}}{i !} \mu_{F, i} \mathrm{D}^{i}
$$

Laplace characters have useful algebraic properties which are described in Barbe and McCormick (2004). In particular, consider the ring $\mathbb{R}_{m}[\mathrm{D}]$ defined as the quotient ring of polynomials in $\mathrm{D}$ with real coefficients modulo the ideal generated by $\mathrm{D}^{m+1}$. Laplace characters are elements of this ring, and can be multiplied. It may be helpful to think of a Laplace character as a formal Laplace transform $\mathrm{E}^{-X \mathrm{D}}$, where $X$ has distribution $F$, expressed as a formal Taylor series in $\mathrm{D}$, dropping all terms in $\mathrm{D}^{m+1}, \mathrm{D}^{m+2}, \ldots$. Then, the multiplication in the ring $\mathbb{R}_{m}[\mathrm{D}]$ amounts to the usual multiplication of Taylor series, dropping any term in $\mathrm{D}^{m+1}, \mathrm{D}^{m+2}, \ldots$. In particular, in $\mathbb{R}_{m}$ [D], we have $L_{H \star K, m}=L_{H, m} L_{K, m}$. In what follows, we always consider Laplace characters of order $m$ as members of $\mathbb{R}_{m}[\mathrm{D}]$, and all the operations on Laplace characters are in that quotient ring.

The following theorem, which we prove in Section 5, provides an asymptotic expansion for the tail of $G$. A less elegant but more explicit formulation is given as a corollary.

Theorem 2.1. Let $F$ be a distribution function whose hazard rate is smoothly varying with negative index at least -1 and positive order $m$. Assume, further, that (2.2) holds and that the moment generating function of $N$ is finite in a neighborhood of the origin. Then, for any nonnegative integer $k \leq m$ for which $F$ admits a moment of order $k$, we have

$$
\bar{G}=\mathrm{E} N L_{F^{\star(N-1)}, k} \bar{F}+o\left(h^{k} \bar{F}\right) .
$$


Remark 2.1. It was shown, by Barbe and McCormick (2005, Lemma 4.1.1), that under the assumptions of Theorem 2.1 the asymptotic equivalence $\bar{F}^{(k)} \sim(-1)^{k} h^{k} \bar{F}$ holds. Therefore, the remainder term in the above formula could be written as $o\left(\bar{F}^{(k)}\right)$.

Remark 2.2. In the statement of Theorem 2.1, the assumption that $F$ admits a moment of order $k$ has bearing only on the lower tail of the distribution for $\bar{F}$ is rapidly varying.

We will see in the next section that the formulation of Theorem 2.1 is quite adequate for practical calculations. A more explicit formulation may be interesting both for understanding the meaning of Theorem 2.1 and for further theoretical developments. To write down such a formulation, we introduce the factorial moments of $N$; writing $(N)_{p}=N(N-1) \cdots(N-p+1)$ for the falling factorial of order $p$ of $N$, the $p$ th factorial moment of $N$ is $\mathrm{E}(N)_{p}$. Given a multiindex $k=\left(k_{1}, \ldots, k_{n}\right)$ of nonnegative integers and extending the classical notation for the multinomial coefficients, we write

$$
\left(\begin{array}{l}
n \\
k
\end{array}\right) \text { or }\left(\begin{array}{c}
n \\
k_{1} \cdots k_{n}
\end{array}\right)
$$

for $n ! / k_{1} ! \cdots k_{n}$ !, and the norm $|k|=k_{1}+\cdots+k_{n}$. We define the following coefficients, involving only the moments of $F$ and the factorial moments of $N$ : when $i=0$, we set $g_{0}=\mathrm{E} N$ and, for any positive $i$, we have

$$
\begin{aligned}
g_{i} & =\frac{(-1)^{i}}{i !} \sum_{\substack{k_{1}+2 k_{2}+\cdots+i k_{i}=i \\
k_{1}, \ldots, k_{i} \geq 0}}\left(\begin{array}{l}
i \\
k
\end{array}\right) \mathrm{E}(N)_{|k|+1}\left(\frac{\mu_{F, 1}}{1 !}\right)^{k_{1}} \cdots\left(\frac{\mu_{F, i}}{i !}\right)^{k_{i}} \\
& =\frac{(-1)^{i}}{i !} \sum_{1 \leq p \leq i} \frac{\mathrm{E}(N)_{p+1}}{p !} \sum_{\substack{j_{1}+\cdots+j_{p}=i \\
j_{1}, \ldots, j_{p}>0}}\left(\begin{array}{c}
i \\
j_{1} \cdots j_{p}
\end{array}\right) \mu_{F, j_{1}} \cdots \mu_{F, j_{p}} .
\end{aligned}
$$

The following corollary asserts that $\bar{G}$ has an asymptotic expansion in the natural asymptotic scale $\left(\bar{F}^{(i)}\right)_{0 \leq i \leq m}$, whose coefficients are given by the $g_{i}$.

Corollary 2.1. Under the assumptions of Theorem 2.1, we have

$$
\bar{G}=\sum_{0 \leq i \leq m} g_{i} \bar{F}^{(i)}+o\left(h^{m} \bar{F}\right) .
$$

Proof. Consider the probability generating function $\Lambda(t)=\mathrm{E} t^{N}$ of $N$ and the Laplace transform $\mathcal{L}(t)=\mathrm{Ee}^{-t X}$ of $X$. Considered as an element in $\mathbb{R}_{m}[\mathrm{D}]$, the operator $\mathcal{L}(\mathrm{D})$ coincides with the Laplace character $L_{F, m}$. Consequently, introducing the derivative $\Lambda^{\prime}$ of $\Lambda$, in $\mathbb{R}_{m}[\mathrm{D}]$ we have

$$
\mathrm{E} N L_{F^{\star(N-1)}, m}=\mathrm{E} N L_{F, m}^{N-1}=\Lambda^{\prime}(\mathcal{L}(\mathrm{D}))
$$

Given Theorem 2.1, it suffices to evaluate the right-hand side of this equality. For this purpose, consider the function defined in a neighborhood of the origin of the real line $g(t)=\Lambda^{\prime}(\mathcal{L}(t))$. This function has a Taylor expansion $\sum_{i>0} t^{i} g^{(i)}(0) / i$ !. Applying the Faà di Bruno formula (see, e.g. Roman (1980)), we see that $g^{(i)}(0)=i ! g_{i}$. The result follows. 


\section{Examples}

We illustrate the use of Theorem 2.1, considering the cases where $N$ has a Poisson and a geometric distribution.

\subsection{Example 1}

Assume that $N$ has a Poisson distribution with parameter $a$. Sums with a Poisson number of summands are commonly used in insurance mathematics, modeling total claim size; see Beirlant et al. (1996), Embrechts et al. (1997), and Willmot and Lin (2001). The following expansion is easily derived.

Proposition 3.1. Let $F$ be a distribution function satisfying the assumptions of Theorem 2.1. If $N$ has a Poisson distribution with parameter $a$, then $\bar{G}=a L_{G, m} \bar{F}+o\left(h^{m} \bar{F}\right)$. Moreover, $L_{G, m}=\exp \left(a\left(L_{F, m}-\mathrm{Id}\right)\right)$.

Proof. Combine Theorem 2.1 and the proof of Corollary 4.4.2 of Barbe and McCormick (2004) to obtain the expansion $a L_{G, m} \bar{F}$. To obtain the expression for $L_{G, m}$, write, in the quotient ring,

$$
\mathrm{E} N L_{F, m}^{N-1}=\mathrm{e}^{-a} \sum_{n \geq 1} n \frac{a^{n}}{n !} L_{F, m}^{n-1}=a \exp \left(a\left(L_{F, m}-\mathrm{Id}\right)\right) .
$$

The above formula is easily implemented with a computer algebra system. For example, the following MAPLE ${ }^{\circledR}$ code calculates $a \exp \left(a\left(L_{F, m}-\mathrm{Id}\right)\right)$ :

$\operatorname{mu}[0]:=1:$

$L F:=\operatorname{sum}\left({ }^{\prime}(-1)^{\wedge} j * m u[j] * D^{\wedge} j / j !{ }^{\prime},{ }^{\prime} j^{\prime}=0 \ldots m+1\right):$

taylor $\left(a * \exp \left(a^{*}(L F-1)\right), D=0, m+1\right)$;

Setting $m=3$ in the above code yields the following first four terms:

$$
\begin{aligned}
\mathrm{E}\left(N L_{F, 3}^{N-1}\right)= & a \mathrm{Id}-a^{2} \mu_{F, 1} \mathrm{D}+\frac{a^{2}}{2}\left(a \mu_{F, 1}^{2}+\mu_{F, 2}\right) \mathrm{D}^{2} \\
& -\frac{a^{2}}{6}\left(a^{2} \mu_{F, 1}^{3}+3 a \mu_{F, 1} \mu_{F, 2}+\mu_{F, 3}\right) \mathrm{D}^{3} .
\end{aligned}
$$

To give a very concrete example, assume that $\bar{F}$ is the Weibull distribution with parameter $\frac{1}{3}$, so that $\bar{F}(t)=\exp \left(-t^{1 / 3}\right)$. Define $e_{r}(t)=t^{r} \exp \left(-t^{1 / 3}\right)$. We obtain, after evaluation of $\mathrm{E}\left(N L_{F, 3}^{N-1}\right)$, and using a computer algebra package,

$$
\begin{aligned}
\bar{G}= & a e_{0}+2 a^{2} e_{-2 / 3}+2 a^{2}(20+a) e_{-4 / 3}+4 a^{2}(20+a) e_{-5 / 3} \\
& +\frac{4 a^{2}\left(1680+60 a+a^{2}\right)}{3} e_{-2}+8 a^{2}\left(1680+60 a+a^{2}\right) e_{-7 / 3} \\
& +\frac{2 a^{2}\left(403200+9120 a+140 a^{2}+a^{3}\right)}{3} e_{-8 / 3}+o\left(e_{-8 / 3}\right) .
\end{aligned}
$$

Perhaps the only remarkable feature of such a seven-term expansion is that it can be done. 


\subsection{Example 2}

Motivated by applications to queueing theory (see, e.g. Cohen (1972) or Bingham et al. (1989, p. 387)), consider the case where $N$ has a geometric distribution with parameter $a$, that is, $N$ is a nonnegative integer $n$ with probability $(1-a) a^{n}$. Again, Theorem 2.1 provides a compact expression of the asymptotic expansion of $\bar{G}$, and the issue is how to actually compute it.

Any polynomial in $\mathrm{D}$ with nonvanishing constant term is invertible in the quotient ring $\mathbb{R}_{m}[\mathrm{D}]$. Therefore, since $a$ is positive and less than 1 , we have

$$
\mathrm{E} N L_{F^{\star(N-1)}, m}=(1-a) \sum_{n \geq 1} a^{n} n L_{F, m}^{n-1}=a(1-a)\left(\mathrm{Id}-a L_{F, m}\right)^{-2} .
$$

Consequently, the following result holds.

Proposition 3.2. Let $F$ be a distribution function satisfying the assumptions of Theorem 2.1. If $N$ has a geometric distribution with parameter $a$, then $\bar{G}=a(1-a)\left(\operatorname{Id}-a L_{F, m}\right)^{-2} \bar{F}+o\left(h^{m} \bar{F}\right)$.

Setting $m=3$, we obtain, as in the previous example, with the help of a computer algebra package, with $b=a /(1-a)$,

$$
\begin{aligned}
\mathrm{E} N L_{F, 3}^{N-1}= & b \mathrm{Id}-2 b^{2} \mu_{F, 1} \mathrm{D}+b^{2}\left(\mu_{F, 2}+3 b \mu_{F, 1}^{2}\right) \mathrm{D}^{2} \\
& -\frac{b^{2}}{3}\left(12 b^{2} \mu_{F, 1}^{3}+9 b \mu_{F, 1} \mu_{F, 2}+\mu_{F, 3}\right) \mathrm{D}^{3} .
\end{aligned}
$$

For instance, when $F$ is the Weibull distribution with parameter $\frac{1}{3}$, the calculation of E $N L_{F, 3}^{N-1}$ yields the following seven-term expansion, where $e_{r}(t)=t^{r} \exp \left(-t^{1 / 3}\right)$ (expressed solely with $a$, the formula contains alternating signs; expressing it with $b=a /(1-a)$ makes it slightly more stable numerically):

$$
\begin{aligned}
\bar{G}= & b e_{0}+4 b^{2} e_{-2 / 3}+4 b^{2}(20+3 b) e_{-4 / 3}+8 b^{2}(20+3 b) e_{-5 / 3} \\
& +32 b^{2}\left(140+15 b+b^{2}\right) e_{-2}+192 b^{2}\left(140+15 b+b^{2}\right) e_{-7 / 3} \\
& +80 b^{2}\left(6720+456 b+28 b^{2}+b^{3}\right) e_{-8 / 3}+o\left(e_{-8 / 3}\right) .
\end{aligned}
$$

\section{Concluding remarks}

In a different subexponential context, namely assuming that $\bar{F}$ is regularly varying, Mikosch and Nagaev (2001) produced an example showing that the rate of convergence in the firstorder asymptotic approximation $\bar{G} \sim \mathrm{E} N \bar{F}$ can be arbitrarily slow, showing that the use of this approximation can be problematic. However, they showed that smoothness of $\bar{F}$, meaning essentially here that it admits a regularly varying density, ensures that the relative error in the approximation decays in the same way as $1 /$ Id. Along the same lines, Barbe and McCormick (2004) obtained higher-order terms yielding, in theory, a more precise approximation under a higher-order regularity condition. We could therefore believe that the key to prevent an arbitrarily slow rate of convergence is smoothness of the distribution. Interestingly, Theorem 2.1 shows that a problem may arise in another direction, when the distribution becomes too close to an exponential one, yet being subexponential. Indeed, considering $m=1$ in Theorem 2.1, we see that, in $\mathbb{R}_{1}[\mathrm{D}]$,

$$
L_{F^{\star(N-1)}, 1}=L_{F, 1}^{N-1}=\left(\mathrm{Id}-\mu_{F, 1} \mathrm{D}\right)^{N-1}=\mathrm{Id}-(N-1) \mu_{F, 1} \mathrm{D},
$$


so that

$$
\begin{aligned}
\bar{G} & =\mathrm{E} N \bar{F}-\mathrm{E} N(N-1) \mu_{F, 1} \bar{F}^{\prime}+o(h \bar{F}) \\
& =\mathrm{E} N \bar{F}+\operatorname{E~} N(N-1) \mu_{F, 1} h \bar{F}+o(h \bar{F}) .
\end{aligned}
$$

Therefore, the relative error of the first-order term is

$$
\frac{\bar{G}-\mathrm{E} N \bar{F}}{\mathrm{E} N \bar{F}} \sim \frac{\mathrm{E} N(N-1)}{\mathrm{E} N} \mu_{F, 1} h .
$$

Note that there is no restriction on the rate of convergence of the hazard rate to 0 besides (2.1) and (2.2), and that this allows for arbitrary slow polynomial rates - it is conceivable that a more refined analysis would show that an arbitrarily slow rate can be obtained by taking distributions even closer to the exponential one. To fix the ideas, if $h(t) \sim t^{-\alpha}$ with $0<\alpha<1$, then

$$
\bar{F}(t)=\exp \left(-\frac{t^{1-\alpha}}{1-\alpha}(1+o(1))\right) .
$$

Hence, arbitrarily slow polynomial rates can be obtained for a distribution with $\alpha$ close to 0 , that is, a distribution close to the exponential distribution. However, again, smoothness and integrability may rescue a poor rate in allowing a higher-order expansion by choosing $m$ large in Theorem 2.1.

\section{Proof of Theorem 2.1}

When $m$ vanishes, Theorem 2.1 is due to Embrechts et al. (1979, p. 342). Therefore, we will prove it when $m$ is at least 1 . The proof is carried out in two steps. We first prove Theorem 2.1 under the extra assumption that the distribution function $F$ is concentrated on the nonnegative half-line; in the second part of the proof, we remove this assumption.

\subsection{Proof in the nonnegative case}

Until further notice, we assume that $F$ is concentrated on the nonnegative half-line. It is convenient to introduce a pseudo-semi-norm on tails. If $K$ is a distribution function, we write

$$
|\bar{K}|_{F}=\sup _{t \geq 0}\left(\frac{\bar{K}}{\bar{F}}\right)(t),
$$

with the convention $0 / 0=0$. This generates balls $B(F, r)$ containing all tails $\bar{K}$ which are less than $r \bar{F}$. We write $B(F)$ for the union of all these balls for all positive $r$. Also, we write $G_{n}$ for the $n$-fold convolution $F^{\star n}$.

We start by recalling Kesten's global bound on the tail function of self-convolutions of subexponential distributions; see Athreya and Ney (1972, Section IV.4, Lemma 7). This result asserts that for any positive $\varepsilon$ there exists a positive $A$ such that, for all positive integers $n$,

$$
\left|\bar{G}_{n}\right|_{F} \leq A(1+\varepsilon)^{n}
$$

We also need a precise estimate of the order of magnitude of derivatives of $\bar{F}$. As noted in Remark 2.1, Lemma 4.1.1 of Barbe and McCormick (2005) shows that, for any nonnegative $k \leq m$, we have

$$
\bar{F}^{(k)} \sim(-1)^{k} h^{k} \bar{F}
$$


Finally, we also need a basic representation of convolution in terms of operators. For any distribution function $K$ with support in the nonnegative half-line and any $\eta$ positive and less than 1 , define the operator

$$
T_{K, \eta} f(t)=\int_{0}^{\eta t} f(t-x) \mathrm{d} K(x) .
$$

For any positive $c$, we also define the multiplication operator $M_{c}$ acting on functions by

$$
M_{c} f(t)=f\left(\frac{t}{c}\right)
$$

These two operators allow us to write a convolution in a way suitable for our analysis. Define the powers $T_{K, \eta}^{n}$ by $T_{K, \eta}^{0}=\mathrm{Id}$ and $T_{K, \eta}^{n+1}=T_{K, \eta} T_{K, \eta}^{n}$. Using proposition 5.1.1 of Barbe and McCormick (2004) inductively, we obtain the following representation for the distribution function, valid on the nonnegative half-line:

$$
\bar{G}_{n}=\sum_{1 \leq i \leq n} T_{F, \eta}^{i-1} T_{G_{n-i}, 1-\eta} \bar{F}+\sum_{1 \leq i \leq n} T_{F, \eta}^{i-1}\left(M_{1 / \eta} \bar{F} M_{1 /(1-\eta)} \bar{G}_{n-i}\right) .
$$

Our first lemma is a simple moment bound.

Lemma 5.1. Let $i$ be a nonnegative integer, and let $\varepsilon$ be a positive real number. There exists $t_{1}$ such that, for any $t \geq t_{1}$ and any distribution function $K$ in $B(F)$, we have

$$
\int_{t}^{\infty} x^{i} \mathrm{~d} K(x) \leq(1+\varepsilon)|\bar{K}|_{F} t^{i} \bar{F}(t) .
$$

Proof. For any nonnegative integer $i$, integration by parts yields

$$
\int_{t}^{\infty} x^{i} \mathrm{~d} K(x)=t^{i} \bar{K}(t)+i \int_{t}^{\infty} x^{i-1} \bar{K}(x) \mathrm{d} x .
$$

The right-hand side of this equality is less than $|\bar{K}|_{F}$ times the same expression with $K$ replaced by $F$. Consequently, it suffices to prove the result when $K=F$. In this case, let $M$ be a positive real number so that $\varepsilon(M-i) \geq i$. Since Id $h$ tends to infinity at infinity, $h$ is more than $M / \mathrm{Id}$ ultimately. For any large enough $t$ and any $x \geq t$, we have

$$
\frac{\bar{F}(x)}{\bar{F}(t)}=\exp \left(-\int_{t}^{x} h(u) \mathrm{d} u\right) \leq\left(\frac{t}{x}\right)^{M} .
$$

This implies that the integral on the right-hand side of (5.4), when $F$ is substituted for $K$, is at most $\varepsilon t^{i} \bar{F}(t)$.

Our next lemma contains the main argument of the proof, namely that a $T_{K, \eta}$ operator is in some sense very close to a Laplace character as far as tail behavior is concerned when applied to $\bar{F}$ and its derivatives.

Lemma 5.2. For any fixed integer $p \leq m$,

$$
\lim _{t \rightarrow \infty} \sup _{K \in B(F)} \frac{\left|\left(T_{K, \eta}-L_{K, m-p}\right) \bar{F}^{(p)}\right|}{|\bar{K}|_{F} h^{m} \bar{F}}(t)=0 .
$$


Proof. The proof of Lemma 4.2.3 of Barbe and McCormick (2005) shows that, for any positive $\delta$,

$$
\left|\int_{0}^{\delta / h(t)} \bar{F}^{(p)}(t-x) \mathrm{d} K(x)-L_{K, m-p} \bar{F}^{(p)}\right|
$$

is at most

$$
\begin{aligned}
& \sum_{0 \leq j \leq m-p}\left|\bar{F}^{(p+j)}(t)\right| \int_{\delta / h(t)}^{\infty} x^{j} \mathrm{~d} K(x) \\
& \quad+\int_{0}^{\delta / h(t)} \int_{0}^{x} \frac{y^{m-p-1}}{(m-p-1) !}\left|\bar{F}^{(m)}(t-x+y)-\bar{F}^{(m)}(t)\right| \mathrm{d} y \mathrm{~d} K(x) .
\end{aligned}
$$

Let $\varepsilon$ be a positive number. Using Lemma 5.1 and (5.2), we see that, for large $t$, the term in (5.6) is less than

$$
\bar{F}(t) 2|\bar{K}|_{F}\left(\frac{\delta}{h(t)}\right)^{j} \bar{F}\left(\frac{\delta}{h(t)}\right) .
$$

Since $\bar{F}$ is rapidly varying, this is ultimately less than $\varepsilon|\bar{K}|_{F} h^{m} \bar{F}$.

The proof of Lemma 4.2.3 of Barbe and McCormick (2005) shows that, for small enough $\delta$, for any large enough $t$, and for any $K$ in $B(F)$, the double integral (5.7) is at most $\varepsilon|\bar{K}|_{F} \mu_{F, m-p} h^{m} \bar{F}$. Hence, we have shown that (5.5) is at most $\varepsilon|\bar{K}|_{F}\left(\mu_{F, m-p}+1\right) h^{m} \bar{F}$ ultimately uniformly over $B(F)$.

The proof of Lemma 4.2.4 of Barbe and McCormick (2005) shows that, for any positive $\delta$ and $\eta$, ultimately uniformly over $B(F)$,

$$
\int_{\delta / h(t)}^{\eta t}\left|\bar{F}^{(p)}(t-x)\right| \mathrm{d} K(x) \leq \varepsilon|\bar{K}|_{F} h^{m} \bar{F}(t) .
$$

This completes the proof.

Lemma 5.2 yields the following estimate on an operator $T$ composed with a Laplace character applied to a derivative of $\bar{F}$.

Lemma 5.3. The following uniform limit holds:

$$
\lim _{t \rightarrow \infty} \sup _{\substack{K \in B(F) \\ H: \mu_{H, m-p}<\infty}} \frac{\left|T_{K, \eta} L_{H, m-p} \bar{F}^{(p)}-L_{K \star H, m-p} \bar{F}^{(p)}\right|}{|\bar{K}|_{F} h^{m} \bar{F} \sum_{0 \leq j \leq m-p}\left(\mu_{H, j} / j !\right)}(t)=0 .
$$

Proof. Since $T_{K, \eta}$ is linear and

$$
L_{H, m-p} \bar{F}^{(p)}=\sum_{0 \leq j \leq m-p} \frac{(-1)^{j}}{j !} \mu_{H, j} \bar{F}^{(p+j)},
$$

the result follows from Lemma 5.2 and Lemma 2.1.4 of Barbe and McCormick (2004).

The next two lemmas will take care of some remainder terms. The first one asserts that terms of order $o\left(h^{m} \bar{F}\right)$ remain so through the action of some $T$ operators. 
Lemma 5.4. Let $q$ be a nonnegative integer and $\varepsilon$ be a positive real number. There exist $t_{2}$ and some positive $A$ and $\eta$ such that, for any positive integer $i$,

$$
T_{F, \eta}^{i}\left(h^{q} \bar{F}\right) \leq A(1+\varepsilon)^{i} h^{q} \bar{F}
$$

on $\left[t_{2}, \infty\right)$.

Proof. Let $\varepsilon$ be a positive real number. Since $h$ is regularly varying with negative index, provided that $\eta$ is small enough, we have $h(t-x) \leq(1+\varepsilon) h(t)$ for any large enough $t$ and any nonnegative $x \leq \eta t$. Therefore, for $t \geq t_{2}^{\prime}$,

$$
\begin{aligned}
T_{F, \eta}\left(h^{q} \bar{F}\right)(t) & =\int_{0}^{\eta t}\left(h^{q} \bar{F}\right)(t-x) \mathrm{d} F(x) \\
& \leq(1+\varepsilon) h^{q}(t) \int_{0}^{\eta t} \bar{F}(t-x) \mathrm{d} F(x) \\
& \leq(1+\varepsilon) h^{q}(t) \overline{F^{\star 2}}(t) .
\end{aligned}
$$

By induction, it follows that

$$
T_{F, \eta}^{i}\left(h^{q} \bar{F}\right)(t) \leq(1+\varepsilon)^{i}\left(h^{q} \overline{F^{\star(i+1)}}\right)(t) .
$$

Using Kesten's bound, (5.1), this shows that $T_{F, \eta}^{i}\left(h^{q} \bar{F}\right)$ is ultimately at most $A(1+\varepsilon)^{2 i} h^{q} \bar{F}$, which completes the proof since $\varepsilon$ is arbitrary.

Our penultimate lemma will be used to handle the terms involving the multiplication operators in (5.3).

Lemma 5.5. Let $\varepsilon$ be a positive real number. There exists $t_{3}$ such that, for any positive integers $i$ and $m$,

$$
\left|M_{1 / \eta} \bar{F} M_{1 /(1-\eta)} \bar{G}_{i}\right| \leq(1+\varepsilon)^{i} h^{m+1} \bar{F}
$$

on $\left[t_{3}, \infty\right)$.

Proof. Kesten's bound, (5.1), shows that

$$
\left|\bar{F}(t \eta) \bar{G}_{i}(t(1-\eta))\right| \leq \bar{F}(t \eta) A(1+\varepsilon)^{i} \bar{F}(t(1-\eta))
$$

Arguing as in Lemma 4.2.1 of Barbe and McCormick (2005), we find that $\bar{F}(t \eta) \bar{F}(t(1-\eta))$ is $o\left(h^{q} \bar{F}(t)\right)$ for any positive $q$. This implies the result.

Our last lemma is stated merely to avoid digression in the argument later on.

Lemma 5.6. Let $\varepsilon$ be a positive number. There exists $A$ such that, for any positive integer $n$,

$$
\sum_{0 \leq j \leq m} \frac{\mu_{G_{n}, j}}{j !} \leq A(1+\varepsilon)^{n} .
$$

Proof. The lemma follows from the Marcinkiewicz-Zygmund inequality (see Chow and Teicher (1988, Section 10.3, Theorem 3)), which implies that $\mu_{G_{n}, j} \leq A n^{j}$ for some constant $A$. 
We can now conclude the proof of Theorem 2.1 under the additional assumption that the distribution function is concentrated on the nonnegative half-line. Combining Lemmas 5.4 and 5.5, there exists an interval $\left[t_{3}, \infty\right)$ on which, for any $j$ and $k$ with $0 \leq j \leq k \leq n$ and for any positive $i$ and $n$ with $i \leq n$,

$$
\left|T_{F, \eta}^{i-1}\left(M_{1 / \eta} \bar{F} M_{1 /(1-\eta)} \bar{G}_{n-i}\right)\right| \leq A(1+\varepsilon)^{n} h^{m+1} \bar{F} .
$$

Equation (5.3) yields, on $\left[t_{3}, \infty\right)$,

$$
\left|\bar{G}_{n}-\sum_{1 \leq i \leq n} T_{F, \eta}^{i-1} T_{G_{n-i, 1-\eta}} \bar{F}\right| \leq A n(1+\varepsilon)^{n} h^{m+1} \bar{F} .
$$

Let $\varepsilon$ be a positive real number, small enough so that $\mathrm{E}(1+2 \varepsilon)^{N}$ is finite. Let $\delta$ be a positive real number. Combining Lemmas 5.2, 5.3, 5.4, and 5.6 and also using Kesten's bound, ultimately, uniformly in $n$ and $i \leq n$, we have

$$
\begin{aligned}
\mid T_{F, \eta}^{i-1} & T_{G_{n-i}, 1-\eta} \bar{F}-T_{F, \eta}^{i-2} L_{G_{n-i+1}, m} \bar{F} \mid \\
& \leq T_{F, \eta}^{i-1}\left|\left(T_{G_{n-i}, 1-\eta}-L_{G_{n-i}, m}\right) \bar{F}\right|+T_{F, \eta}^{i-2}\left|\left(T_{F, \eta} L_{G_{n-i}, m}-L_{G_{n-i+1, m}}\right) \bar{F}\right| \\
& \leq 2 \delta\left(A^{2}+A\right)(1+\varepsilon)^{n} h^{m} \bar{F} .
\end{aligned}
$$

Using the same combination of lemmas, we also have, ultimately, uniformly in $n$ and $j \leq n-1$,

$$
\left|T_{F, \eta}^{j} L_{G_{n-j-1}, m} \bar{F}-T_{F, \eta}^{j-1} L_{G_{n-j}, m} \bar{F}\right| \leq A^{2}(1+\varepsilon)^{n} \delta h^{m} \bar{F}
$$

We take $A>1$, simply to ensure that $A^{2}$ is more than $A$. Summing (5.10) for $j$ positive and less than $i$ and adding (5.9), we obtain

$$
\left|T_{F, \eta}^{i-1} T_{G_{n-i}, 1-\eta} \bar{F}-L_{G_{n-1}, m} \bar{F}\right| \leq 4 A^{2} \delta h^{m} \bar{F} i(1+\varepsilon)^{n}
$$

on some interval $\left[t_{4}, \infty\right)$. Summing these inequalities for positive $i \leq n$ and combining with (5.8) yields

$$
\left|\bar{G}_{n}-n L_{G_{n-1}, m} \bar{F}\right| \leq 10 A^{2} \delta n(n+1)(1+\varepsilon)^{n} h^{m} \bar{F} .
$$

Since the moment generating function of $N$ is finite at $\log (1+2 \varepsilon)$ and $\delta$ is arbitrary, Theorem 2.1 follows for distributions satisfying the hypothesis of the theorem and concentrated on the nonnegative half-line.

\subsection{Removing the nonnegativity assumption}

Now that we have proved Theorem 2.1 for nonnegative random variables, we extend the result by removing the nonnegativity assumption. To make such an extension nontrivial, from now on we assume, without any loss of generality, that $F(0-)$ is positive and $F(0)<1$.

Let $1-p$ be $F(0-)$. Let $F_{+}$and $F_{-}$be the distribution functions respectively defined on the nonnegative and negative half-lines by $F_{-}=F /(1-p)$ and $F_{+}=(F-(1-p)) / p$. We see that $F=(1-p) F_{-}+p F_{+}$. It follows that, for any nonnegative integer $n$,

$$
\overline{F^{\star n}}=\sum_{0 \leq k \leq n}\left(\begin{array}{l}
n \\
k
\end{array}\right) p^{k}(1-p)^{n-k} \overline{F_{+}^{\star k} \star F_{-}^{\star(n-k)}} .
$$


This expression involves convolutions $F_{+}^{\star k} \star F_{-}^{\star(n-k)}$ of two distribution functions concentrated on the nonnegative and negative half-lines, respectively. To any distribution function $H$ concentrated on the negative half-line, we associate the operator $U_{H}$ defined by

$$
U_{H} f(t)=\int_{-\infty}^{0} f(t-x) \mathrm{d} H(x) .
$$

If $K$ is a distribution function on the nonnegative half-line, then $\overline{H \star K}=U_{H} \bar{K}$ on the nonnegative half-line.

The following result is stated mostly for clarity of further arguments so that it can be referred to. Its proof follows directly from the definition of $U_{H}$.

Lemma 5.7. Let a be a positive real number. If $f$ is a nonnegative function nonincreasing on $[a, \infty)$ then, for any distribution function $H$ on the negative half-line, $U_{H} f \leq f$ on $[a, \infty)$.

In particular, this lemma will apply to functions of the form $h^{m} \bar{F}$ because the following result holds.

Lemma 5.8. For any nonnegative integer $m$ the function $h^{m} \bar{F}$ is ultimately nonincreasing.

Proof. The derivative of $h^{m} \bar{F}$ is

$$
\frac{h^{m} \bar{F}}{\operatorname{Id}}\left(m \frac{\operatorname{Id} h^{\prime}}{h}-\operatorname{Id} h\right) .
$$

Since $h$ is smoothly varying of index $\alpha$ and order at least 1 , the function $\operatorname{Id} h^{\prime} / h$ tends to $\alpha$ at infinity. Hence, assumption (2.1) implies that the derivative of $h^{m} \bar{F}$ is ultimately negative.

Our next lemma approximates an operator $U_{H}$ by the Laplace character $L_{H, m}$ when acting on derivatives of $\bar{F}$.

Lemma 5.9. Let $p$ be a nonnegative integer such that $p \leq m$ and let $\varepsilon$ be a positive real number. There exists a neighborhood of $\infty$ such that, for any distribution function $H$ concentrated on the nonpositive half-line and having a finite absolute moment of order $r$ greater than $m$, we have

$$
\left|U_{H} \bar{F}^{(p)}-L_{H, m-p} \bar{F}^{(p)}\right| \leq \varepsilon h^{m} \bar{F} \max _{0 \leq s \leq r} \mu_{M_{-1} H, s} .
$$

Proof. As in Lemma 4.2.3 of Barbe and McCormick (2005), we bound

$$
\left|\int_{-\delta / h(t)}^{0} \bar{F}^{(p)}(t-x) \mathrm{d} H(x)-L_{H, m-p} \bar{F}^{(p)}(t)\right|
$$

by the sum of

$$
\sum_{0 \leq j \leq m-p} \frac{\left|\bar{F}^{(p+j)}(t)\right|}{j !} \int_{-\infty}^{-\delta / h(t)}|x|^{j} \mathrm{~d} H(x)
$$

and

$$
\int_{-\delta / h(t)}^{0}\left|\int_{0}^{x} \frac{y^{m-p-1}}{(m-p-1) !}\left(\bar{F}^{(m)}(t-x+y)-\bar{F}^{(m)}(t)\right) \mathrm{d} y\right| \mathrm{d} H(x) .
$$


Lemma 4.1.1 of Barbe and McCormick (2005) shows that $\left|\bar{F}^{(p+j)}\right| \sim h^{p+j} \bar{F}$ at infinity, while, for $p+j<r$,

$$
\begin{aligned}
\int_{-\infty}^{-\delta / h}|x|^{j} \mathrm{~d} H(x) & =\int_{-\infty}^{-\delta / h}|x|^{p+j-r}|x|^{r-p} \mathrm{~d} H(x) \\
& \leq\left(\frac{\delta}{h}\right)^{p+j-r} \mu_{M_{-1} H, r-p} .
\end{aligned}
$$

Therefore, there exists $t_{5}$, depending only on $F$ and not on $H$, such that (5.13) is at most

$$
2 m\left(h^{r} \bar{F}\right)(t) \delta^{-r} \max _{0 \leq s \leq r} \mu_{M_{-1} H, s}
$$

on $\left[t_{5}, \infty\right)$. Again following the proof of Lemma 4.2.3 of Barbe and McCormick (2005) we see that, for any positive $\varepsilon$, we can fix $\delta$ such that the term (5.14) is bounded by

$$
\varepsilon\left(h^{m} \bar{F}\right)(t) \int_{-\delta / h(t)}^{0}\left|\int_{0}^{x} \frac{y^{m-p-1}}{(m-p-1) !} \mathrm{d} y\right| \mathrm{d} H(x) \leq \varepsilon\left(h^{m} \bar{F}\right)(t) \mu_{M_{-1} H, m-p} .
$$

Finally, using Lemma 5.8 we obtain, provided $t_{5}$ is chosen large enough, for any $t \geq t_{5}$,

$$
\begin{aligned}
\left|\int_{-\infty}^{-\delta / h(t)} \bar{F}^{(p)}(t-x) \mathrm{d} H(x)\right| & \leq 2 \int_{-\infty}^{-\delta / h(t)}\left(h^{p} \bar{F}\right)(t-x) \mathrm{d} H(x) \\
& \leq 2\left(h^{p} \bar{F}\right)(t)\left(\frac{h(t)}{\delta}\right)^{r} \mu_{M_{-1} H, r},
\end{aligned}
$$

and the result follows.

We can now complete the proof of Theorem 2.1. We rewrite (5.12) as

$$
\overline{F^{\star n}}=\sum_{0 \leq k \leq n}\left(\begin{array}{l}
n \\
k
\end{array}\right) p^{k}(1-p)^{n-k} U_{F_{-}^{\star(n-k)}} \overline{F_{+}^{\star k}} .
$$

It follows, from (5.11) and Lemmas 5.7 and 5.8, that there exists a neighborhood of $\infty$ on which, for all nonnegative integers $n$,

$$
\begin{aligned}
\mid \overline{F^{\star n}} & -\sum_{0 \leq k \leq n}\left(\begin{array}{l}
n \\
k
\end{array}\right) p^{k}(1-p)^{n-k} k U_{F_{-}^{\star(n-k)}} L_{F_{+}^{\star(k-1)}, m} \bar{F}_{+} \mid \\
& \leq \sum_{0 \leq k \leq n}\left(\begin{array}{l}
n \\
k
\end{array}\right) p^{k}(1-p)^{n-k} 10 A^{2} \delta k(k+1)(1+\varepsilon)^{k} h^{m} \bar{F} \\
& \leq 20 A^{2} \delta n^{2} \sum_{0 \leq k \leq n}\left(\begin{array}{l}
n \\
k
\end{array}\right) p^{k}(1-p)^{n-k}(1+\varepsilon)^{k} h^{m} \bar{F} \\
& =20 A^{2} \delta n^{2}(1+p \varepsilon)^{n} h^{m} \bar{F} .
\end{aligned}
$$

Hence, by the same argument as we used to conclude the proof of Theorem 2.1 in the nonnegative case, we obtain

$$
\mathrm{E} \overline{F^{\star N}}=\mathrm{E} \sum_{k \geq 1}\left(\mathbf{1}\{k \leq N\}\left(\begin{array}{l}
N \\
k
\end{array}\right) p^{k}(1-p)^{N-k} k U_{F_{-}^{\star(N-k)}} L_{F_{+}^{\star(k-1)}, m} \bar{F}+\right)+o\left(h^{m} \bar{F}\right) .
$$


Lemma 5.9 yields

$$
\begin{aligned}
& \left|U_{F_{-}^{\star(N-k)}} L_{F_{+}^{\star(k-1)}, m} \bar{F}_{+}-L_{F_{-}^{\star(N-k)}, m} L_{F_{+}^{\star(k-1)}, m} \bar{F}_{+}\right| \\
& \leq \varepsilon h^{m} \bar{F} \max _{0 \leq s \leq r} \mu_{M_{-1} F^{\star(N-k),}, \max _{0 \leq s \leq r}} \mu_{F_{+}^{\star(k-1)}, s} .
\end{aligned}
$$

Applying the Marcinkiewicz-Zygmund inequality and possibly redefining $\varepsilon$, this is at most $\varepsilon h^{m} \bar{F} N^{2 r}$. Therefore, since the moment generating function of $N$ is finite in a neighborhood of the origin and $\varepsilon$ is arbitrary, (5.15) implies that

$$
\mathrm{E} \overline{F^{\star N}}=\mathrm{E} \sum_{k \geq 1}\left(\mathbf{1}\{k \leq N\}\left(\begin{array}{l}
N \\
k
\end{array}\right) p^{k}(1-p)^{N-k} k L_{F_{-}^{\star(N-k)}, m} L_{F_{+}^{\star(k-1)}, m} \bar{F}_{+}\right)+o\left(h^{m} \bar{F}\right) .
$$

To complete the proof, note that Laplace characters are linear with respect to their first index, and therefore can be defined for measures. Since

$$
k\left(\begin{array}{l}
N \\
k
\end{array}\right)=N\left(\begin{array}{c}
N-1 \\
k-1
\end{array}\right)
$$

linearity and the morphism property of the Laplace character show that

$$
\sum_{k \geq 1} \mathbf{1}\{k \leq N\}\left(\begin{array}{l}
N \\
k
\end{array}\right) p^{k}(1-p)^{N-k} k L_{F_{-}^{\star(N-k)}, m} L_{F_{+}^{\star(k-1)}, m}
$$

is the Laplace character of order $m$ of

$$
p N \sum_{k \geq 1} \mathbf{1}\{k \leq N\}\left(\begin{array}{c}
N-1 \\
k-1
\end{array}\right) p^{k-1}(1-p)^{N-k} F_{-}^{\star(N-1-(k-1))} \star F_{+}^{\star(k-1)},
$$

that is, the Laplace character of $p N F^{\star(N-1)}$. Applying this Laplace character to $\bar{F}_{+}$and observing that $\bar{F}=p \bar{F}+$ on the nonnegative half-line completes the proof of Theorem 2.1.

\section{Acknowledgement}

The authors thank an anonymous referee whose questions led to a better statement for Theorem 2.1 and to Section 4.

\section{References}

Asmussen, S. (1987). Applied Probability and Queues. John Wiley, Chichester.

Asmussen, S. (1997). Ruin Probabilities. World Scientific, Singapore.

Athreya, K. B. And Ney, P. E. (1972). Branching Processes. Springer, New York.

Barbe, P. AND McCormick, W. P. (2004). Asymptotic expansions for infinite weighted convolutions of heavy tail distributions and applications. To appear in Mem. Amer. Math. Soc.

BArbe, P. AND McCormick, W. P. (2005). Asymptotic expansions for convolutions of light tailed subexponential distributions. To appear in Prob. Theory Relat. Fields.

Beirlant, J., Teugels, J. L. And Vynckier, P. (1996). Practical Analysis of Extreme Values. Leuven University Press.

Beirlant, J., Broniatowski, M., Teugels, J. L. And Vynckier, P. (1995). The mean residual life function at great age: applications to tail estimation. J. Statist. Planning Infer. 45, 21-48.

Bingham, N. H., Goldie, C. M. And Teugels, J. L. (1989). Regular Variation, 2nd edn. Cambridge University Press.

Chow, Y. S. And TeIcher, H. (1988). Probability Theory, Independence, Interchangeability, Martingales, 2nd edn. Springer, New York.

Cline, D. B. H. (1987). Convolutions of distributions with exponential and subexponential tails. J. Austral. Math. Soc. A 43, 347-365. 
Cohen, J. W. (1972). On the tail of the stationary waiting-time distribution and limit theorem for the M/G/1 queue. Ann. Inst. H. Poincaré B 8, 255-263.

DAVIS, R. AND RESNICK, S. (1988). Extremes of moving averages of random variables from the domain of attraction of the double exponential distribution. Stoch. Process. Appl. 30, 41-68.

Embrechts, P. (1985). Subexponential distribution functions and their applications: a review. In Proc. Seventh Conf. Prob. Theory (Braşov, 1982), VNU Science Press, Utrecht, pp. 125-136.

Embrechts, P. And Goldie, C. M. (1982). On convolution tails. Stoch. Process. Appl. 13, 263-278.

Embrechts, P., Goldie, C. M. And VeraverbeKe, N. (1979). Subexponentiality and infinite divisibility. Z. Wahrscheinlichkeitsth. 49, 335-347.

Embrechts, P., KlüPPelberg, C. And Mikosch, T. (1997). Modelling Extremal Events. Springer, Berlin.

Feller, W. (1971). An Introduction to Probability and Its Applications, Vol. II, 2nd edn. John Wiley, New York.

Goldie, C. M. And KlüPpelberg, C. (1998). Subexponential distributions. In A Practical Guide to Heavy Tails, eds R. Adler, R. Feldman, and M. Taqqu, Birkhäuser, Boston, MA, pp. 435-459.

GrüBEL, R. (1987). On subordinated distributions and generalized renewal measures. Ann. Prob. 15, $394-415$.

Mikosch, T. AND NAGAEV, A. (2001). Rates in approximations to ruin probabilities for heavy-tailed distribution. Extremes 4, 67-78.

OMEy, E. AND Willekens, E. (1987). Second-order behaviour of distributions subordinate to a distribution with finite mean. Commun. Statist. Stoch. Models 3, 311-342.

Pakes, A. G. (1975). On the tails of waiting-time distributions. J. Appl. Prob. 12, 555-564.

Roman, S. (1980). The formula of Faà di Bruno. Amer. Math. Monthly 87, 805-809.

Rootzén, H. (1986). Extreme value theory for moving average processes. Ann. Prob. 14, 612-652.

StUart, A. AND Ord, J. K. (1994). Kendall's Advanced Theory of Statistics, Vol. 1, 6th edn. Edward Arnold, London.

Teugels, J. L. (1975). The class of subexponential distributions. Ann. Prob. 3, 1000-1011.

Willmot, G. E. AND Lin, X. S. (2001). Lundberg Approximations for Compound Distributions with Insurance Applications (Lecture Notes Statist. 156). Springer, New York. 